\title{
Plastische Rekonstruktionsmöglichkeiten rund um das Knie
}

Stefan Krischak, Matthias Föhn

\section{Einleitung}

Die Defektdeckung im Bereich des Knies kann eine große Herausforderung darstellen. Es soll zum einen ein dauerhafter und zugleich weicher und flexibler Hautmantel entstehen, zum anderen müssen anatomische und eventuelle infektiologische Besonderheiten beachtet werden. Dieser Artikel soll einen Überblick über die Möglichkeiten der Defektdeckung rund um das Knie geben und einen möglichen Algorithmus aufzeigen.

\section{Ursachen}

Die Gründe, warum es zu einem Defekt im Bereich des Knies kommen kann, sind vielfältig. Neben traumatischen und onkologischen Gründen kann es auch durch chronische Entzündungen und Infektionen zu Weichteildefekten kommen. Auch stellen uns narbige Veränderungen nach Verbrennungen im Kniebereich, aber auch Strahlenschäden vor unterschiedliche Herausforderungen. Um diese meistern zu können, bedarf es einiger Erfahrung, und es sollte auf unterschiedliche Rekonstruktionsverfahren zurückgegriffen werden können.

\section{Relevante Anatomie}

\section{Haut}

Für die Rekonstruktion eines Gewebedefektes im Bereich des Kniegelenkes müssen anatomische Besonderheiten berücksichtigt werden. Im Bereich der Haut ist für eine gute Gelenkfunktion genügend Dehnungsfähigkeit in Beugung und Streckung notwendig. Streckseitig kann es bei einem Hautdefizit zu einer zu großen Spannung mit einer erneuten Wunddehiszenz kommen. Beugeseitig ist bei einer ungenügenden Hautreserve keine vollständige Streckung im Knie mehr möglich. In der Kniekehle müssen zudem die großen Gefäße und Nervenstraßen sicher gedeckt werden. Generell soll das Weichgewebe nicht zu sehr auftragen, da es sonst zu einer sekundären Bewegungseinschränkung kommen kann.

\section{Bursa praepatellaris}

Die Bursa praepatellaris erstreckt sich nahezu über die gesamte Fläche der darunter liegenden Patella. Bereits bei Defekten geringer Tiefe liegen diese Strukturen frei. Nicht selten ist die Infektion der Bursa praepatellaris so- gar die Ursache eines Defektes. Zu beachten ist hier, dass auf dem Bursagewebe die Misserfolgsraten einer Hauttransplantation hoch sind. Nicht selten ist deshalb hier eine Lappendeckung erforderlich.

\section{Kniegelenk}

Relevant für die Defektdeckung sind am Kniegelenk weniger die Kniebinnenstrukturen, sondern insbesondere die äußeren Kapsel-Band-Strukturen. Auf diesen ist eine Hauttransplantation i.d. R. nicht möglich. Zudem ist eine zunehmende Nekrose dieser Strukturen auch bei einer temporären Deckung mit einer Vakuumversiegelung nicht selten. Da direkt unter diesen Strukturen die Gelenkhöhle liegt, ist auch hier bei einem Defekt eine Lappendeckung sinnvoll.

\section{Muskulärer Bewegungsapparat}

Von der umgebenden Muskulatur am Kniegelenk sind insbesondere die ventrale Oberschenkelmuskulatur, vor allem der M. rectus femoris und M. vastus lateralis, und die Wadenmuskulatur, vor allem der M. gastrocnemius, für die Defektdeckung von Bedeutung. Diese haben als mögliche Muskellappen zum einen eine konstante Gefäßversorgung, zum anderen ist die Entnahme von Anteilen dieser Muskeln möglich und hat in vielen Fällen keine wesentlichen Bewegungseinschränkungen zur Folge.

\section{Gefäße und Nerven}

Neben den Bandstrukturen müssen oftmals auch die Gefäß- und Nervenstraßen vor allem im dorsalen und lateralen Kniebereich beachtet und gedeckt werden. An wesentlichen Strukturen sind hier vor allem die A. und V. poplitea im dorsalen Anteil und der N. peronaeus communis im Bereich des Fibulaköpfchens zu nennen. Die Gefäßstrukturen können jedoch auch hinsichtlich der Defektdeckung notwendige Anschlussbereiche darstellen.

\section{Therapieplan}

\section{Management des Wundgrundes}

Vor einer Lappenplastik muss immer eine Säuberung des Wundgrundes erfolgen. Da ohnehin eine Lappendeckung erfolgt, kann dabei Sehnen- und Knochengewebe freigelegt werden. Auf keinen Fall soll ein Lappen auf nekrotisches oder infiziertes Gewebe aufgebracht werden. Das Débridement soll hierbei scharf, z. B. mit einem Skalpell, 

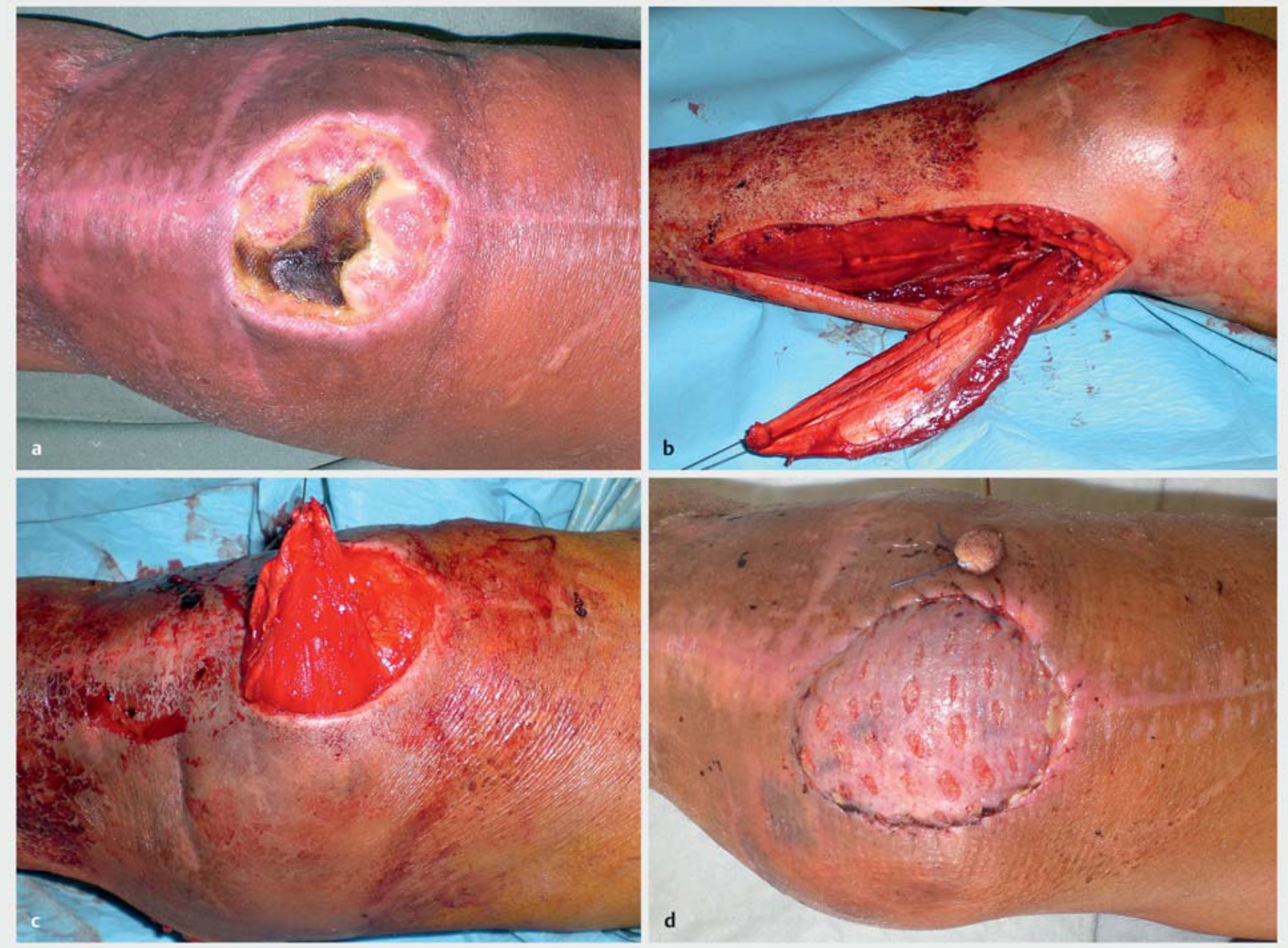

- Abb. 1 a Defekt nach Débridement einer Bursitis praepatellaris. b-d Rekonstruktion mit gestieltem medialen Gastroknemiuslappen und Spalthaut.

erfolgen und nicht nur mit einem scharfen Löffel kürettiert werden. Oberflächlich nekrotische oder angetrocknete Sehnen-, Kapsel- und Bandstrukturen sollten hierbei dünn angefrischt werden, wenn möglich unter Erhalt der Funktion.

\section{Management bei freiliegender Bursa praepatellaris}

Das Bursagewebe vor der Kniescheibe liegt bei einem Defekt im vorderen Kniebereich meist mit frei ( $\sim$ Abb. 1 a). Die Heilungstendenz dieses Gewebes ist i. d. R. sehr gering. Die Einheilung von Spalthaut direkt auf Bursagewebe ist nicht zu erwarten. Ebenso ist die sekundäre Heilung durch offene Wundbehandlung hier im günstigen Fall langwierig. Meist lässt sich hierdurch keine stabile Abheilung erreichen. Auch durch eine längere sog. Vakuumtherapie kommt es nur sehr langsam und in nur geringem Umfang zu einer Bildung von Granulationsgewebe. Eine solche Behandlung sollte entsprechend nur temporär bis zur definitiven Lappendeckung angewendet werden ( $\bullet \mathbf{A b b}$. $\mathbf{1} \mathbf{b}-\mathbf{d}$ ).

\section{Management bei einliegender Knieprothese}

Die prothetische Versorgung mit einer Knieprothese erfolgt i. d. R. durch einen Längsschnitt am ventralen Kniegelenk. Insbesondere auf Höhe des Lig. patellae und präpatellar kommt es durch die dünne Gewebedecke bevorzugt zu Durchblutungsstörungen der Wundränder. Verstärkt wird die Neigung zu Wundheilungsstörungen in diesem Bereich durch eine häufig vermehrte Schwellung und durch die Bewegung des Gelenkes. Im Falle einer Infektion der Knieprothese steigt dieses Risiko zum einen durch die Infektion selbst, aber auch durch die dann meist notwendigen mehrfachen operativen Eingriffe durch den vorhandenen Zugang.

Kann die Prothese durch die Wunde direkt getastet werden oder liegt diese sogar frei, ist das alleinige Débridement des umliegenden Gewebes nicht ausreichend. Hier muss abhängig von einem akuten Frühinfekt oder einem Spätinfekt und abhängig von den mikrobiologischen Ergebnissen zusätzlich ein Austausch der mobilen Teile 
oder ein vollständiger Ausbau der Prothese vorgenommen werden. Bei diesen Patienten ist die Erstellung eines klaren Behandlungsplanes unter Einbeziehung der Orthopädie-Unfallchirurgie, der plastischen Chirurgie und der Mikrobiologie für einen langfristigen Behandlungserfolg unabdingbar. Auch hier soll vor der sog. Vakuumversiegelung als „Rekonstruktionsplan“ ausdrücklich gewarnt werden. Diese allein kann das komplexe Problem nicht lösen. Vielmehr kommt es regelhaft zu einer Fibrosierung des Gewebes und einem Shift des Keimspektrums zu schwer therapierbaren und resistenten Keimen. Bei diesen Patienten sollte daher frühzeitig eine Verlegung in ein entsprechendes Zentrum zur kombinierten Behandlung der keimbesiedelten Prothese und des Gewebedefektes erfolgen.

\section{Management bei freiliegendem Osteosynthesematerial}

Bei Patienten mit Defekten nach Wundheilungsstörungen im Rahmen einer Osteosynthese, z. B. im Bereich der proximalen Tibia, stellt neben dem ggf. freiliegenden Knochen- oder Bandgewebe das sichtbare Osteosynthesematerial eine weitere Indikation zur Lappendeckung dar. Die Grundsätze des o.g. Débridements gelten auch hier. Ob das Metall in einer solchen Situation belassen werden kann oder nicht, hängt von verschiedenen Faktoren ab. Entscheidend ist hierbei die Dauer seit der Implantation und damit die Ausprägung eines sog. bakteriellen Biofilmes. Ist dieser nach einer längeren Besiedelungszeit vorhanden ist mit einer erhöhten Infektionsrate nach einer Abdeckung des Defektes durch einen Lappen zu rechnen.

In einigen Fällen kann bei einer akuten Infektion das Osteosynthesematerial erhalten bleiben. Allerdings sollte hier ein rasches, radikales Débridement von infiziertem Gewebe durchgeführt werden mit bereits frühzeitiger Einplanung einer Lappenplastik ( $\sim$ Abb. 2 a-d). Die antibiotische Nachbehandlung sollte hier ebenfalls in enger Absprache mit einem Mikrobiologen erfolgen.

\section{Defektdeckung}

\section{Hauttransplantation mit oder ohne dermale Ersatzmaterialien (z. B. Matriderm ${ }^{\circledR}$ )}

Die Defektdeckung mit einem Hauttransplantat ist eine häufig verwendete und erfolgversprechende Methode. Im Bereich des Kniegelenkes ist allerdings die Einheilung über der Kniescheibe, trotz einer vitalen Gewebeschicht über dem Knochen, sehr schlecht. Im Bereich der Kniekehle kann es durch eine Spalthaut zu narbigen Kontrakturen und in der Folge zu einem Streckdefizit des Kniegelenkes kommen.

Diese Methode kann aufgrund ihrer geringen Hebemorbidität auch bei schwer kranken Patienten meist problemlos durchgeführt werden und stellt bei oberfläch- lichen Wunden i. d. R. das Mittel der Wahl dar. Wenn dies allerdings nicht zu einer stabilen Deckung führt, sollte dann als nächster Schritt eine Lappenplastik in Erwägung gezogen werden.

Spalthautfähige, großflächige Defekte im Bereich des Kniegelenkes können z.B. nach Décollement-Verletzungen oder nach dem Débridement einer nekrotisierenden Fasziitis verbleiben. Hier kann die Kontrakturneigung durch Dermisersatzmaterialien vermindert werden. Diese können am ventralen und am dorsalen Kniegelenk aufgebracht werden. Die Kosten hierfür sind aufgrund der großen Fläche allerdings sehr hoch. Nach durchgeführter Muskellappenplastik ist die zusätzliche Transplantation von Spalthaut primär oder sekundär eigentlich immer erforderlich.

\section{Lokale Haut-Lappen-Plastiken}

Für örtliche Geweberekonstruktionen kann, bei einer ausreichenden Hautreserve in der Umgebung, eine lokale Hautlappenplastik ausreichend sein. Der u. U. hierbei entstehende Hebedefekt kann mit einem Hauttransplantat gedeckt werden. Aufgrund des meist ohnehin schon grenzwertig durchbluteten Gewebes im Wundrandbereich und im Bereich des perifokalen Ödems eignen sich hierfür lediglich kleine Defekte bis ca. 4 cm Größe. Verlockend ist aufgrund eines häufig bereits vorliegenden langen Längsschnittes am ventralen Knie der Gedanke an einen sog. „Visierlappen“ mit Durchführung eines parallelen Längsschnittes, Ablösen dieser Hautbrücke und Verlagern der Haut über den Defekt. Hierbei muss bedacht werden, dass durch den Gegenschnitt und das Ablösen und die Mobilisation der Haut die Durchblutungssituation in dem Lappen weiter verschlechtert wird. Weiterhin ist zu bedenken, dass die Rotationspunkte des Lappens am proximalen und distalen Ende des Gegenschnittes liegen und dieser damit deutlich länger sein muss als der Defekt. Aus diesen Überlegungen heraus sehen wir in einem Visierlappen keine „sinnvolle“ Option zur Defektdeckung.

Bei Kontrakturen im Bereich der Kniekehle durch längs verlaufende Narbenzüge können diese durch eine (serielle) Z-Plastik i.d.R. problemlos aufgelöst werden.

Zusammenfassend ist die Indikation für örtliche Lappenplastiken im Bereich des Kniegelenkes begrenzt und allenfalls bei kleineren, oberflächlichen Defekten oder Kontrakturen durch Narbenzüge gegeben.

\section{Gefäßgestielte Lappenplastiken mit Haut- und Fettgewebe}

Aus der weiteren Region können Lappenplastiken mit Haut- und Fettgewebe unter Erhalt der Durchblutung gehoben werden. Diese sind sowohl in der Planung als auch in der Präparation deutlich anspruchsvoller. Hierbei muss 

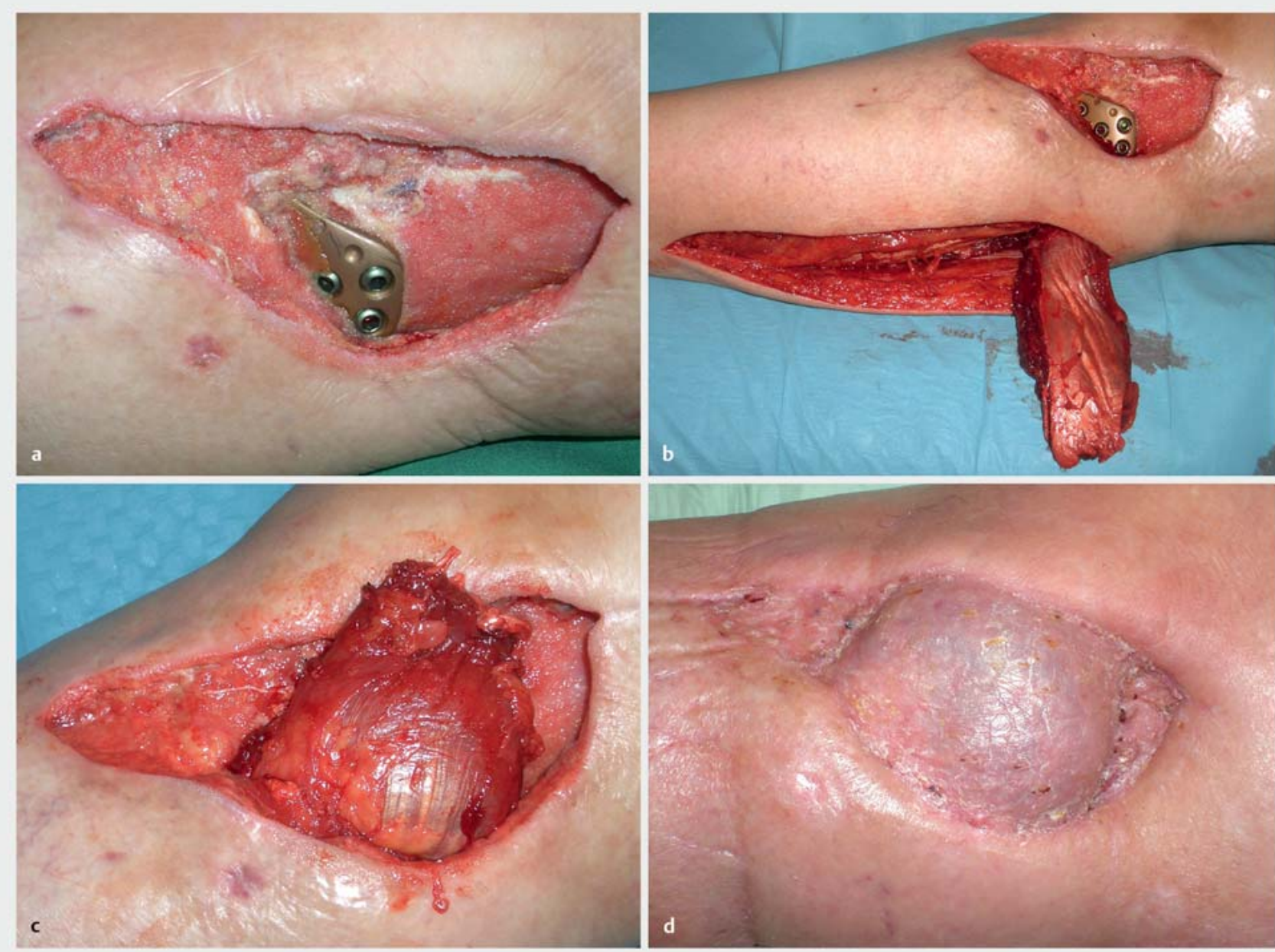

- Abb. 2 a Defekt nach Osteosynthese mit freiliegendem Metall. b-d Rekonstruktion mit gestieltem medialen Gastroknemiuslappen und Spalthaut.

insbesondere die ausreichende Länge des Gefäßstieles und die Lappengröße bei der Planung beachtet werden.

\section{Distal gestielter anterolateraler}

Oberschenkellappen (ALT-Lappen)

Der absteigende Ast der $A$. circumflexa lateralis verläuft zwischen dem M. rectus femoris und dem M. vastus lateralis. Dieser gibt Äste in die Muskulatur ab, von denen wenige die Muskelfaszie perforieren und in die subkutane Ebene gelangen, sog. Perforatoren. Manchmal verlaufen diese Äste auch direkt im Septum zwischen diesen Muskeln. Distal gibt es i.d.R. Anastomosen zwischen dem Hauptgefäß und dem Versorgungsgebiet der A. poplitea. Dies ermöglicht die Hebung eines Lappens mit einem distalen, retrograden Blutfluss. Der Lappen aus Haut- und Fettgewebe wird hierbei um einen Perforator gehoben, das Stielgefäß proximal durchtrennt und nach distal mobilisiert, bis das Gewebe spannungsfrei in den Defekt eingelegt werden kann. Da die Perforatoren selbst und deren Lokalisation sehr variabel ist, sollten diese zuvor mit einer farbcodierten Duplexsonografie lokalisiert und markiert werden. Manchmal sind auch keine Perforatoren oder keine distale Anastomose vorhanden. In diesen Fällen kann der Lappen nicht verwendet werden. Abhängig von der Hautspannung können hier Lappen mit bis zu $20 \mathrm{~cm}$ Länge und $10 \mathrm{~cm}$ Breite entnommen werden. Der entstandene Defekt kann direkt verschlossen werden [1].

\section{Perforatorlappen}

Basierend auf dem Prinzip von Perforatorgefäßen aus der Tiefe in die Haut können sog. „Propellerlappen“ gebildet werden. Hier werden 2 Hautanteile um eine zentrale Gefäßachse eines Perforators umschnitten und um $90^{\circ}$ rotiert. Hierbei wird der größere „Propeller“ in den Defekt eingelegt und der kleinere in den Hebedefekt. Die Wunden können damit alle wieder direkt verschlossen werden. Vorbereitend ist hier immer ein Duplexultraschall oder eine CT-Angiografie erforderlich. Erreicht werden können hiermit vor allem Defekte im distalen Kniebereich. 

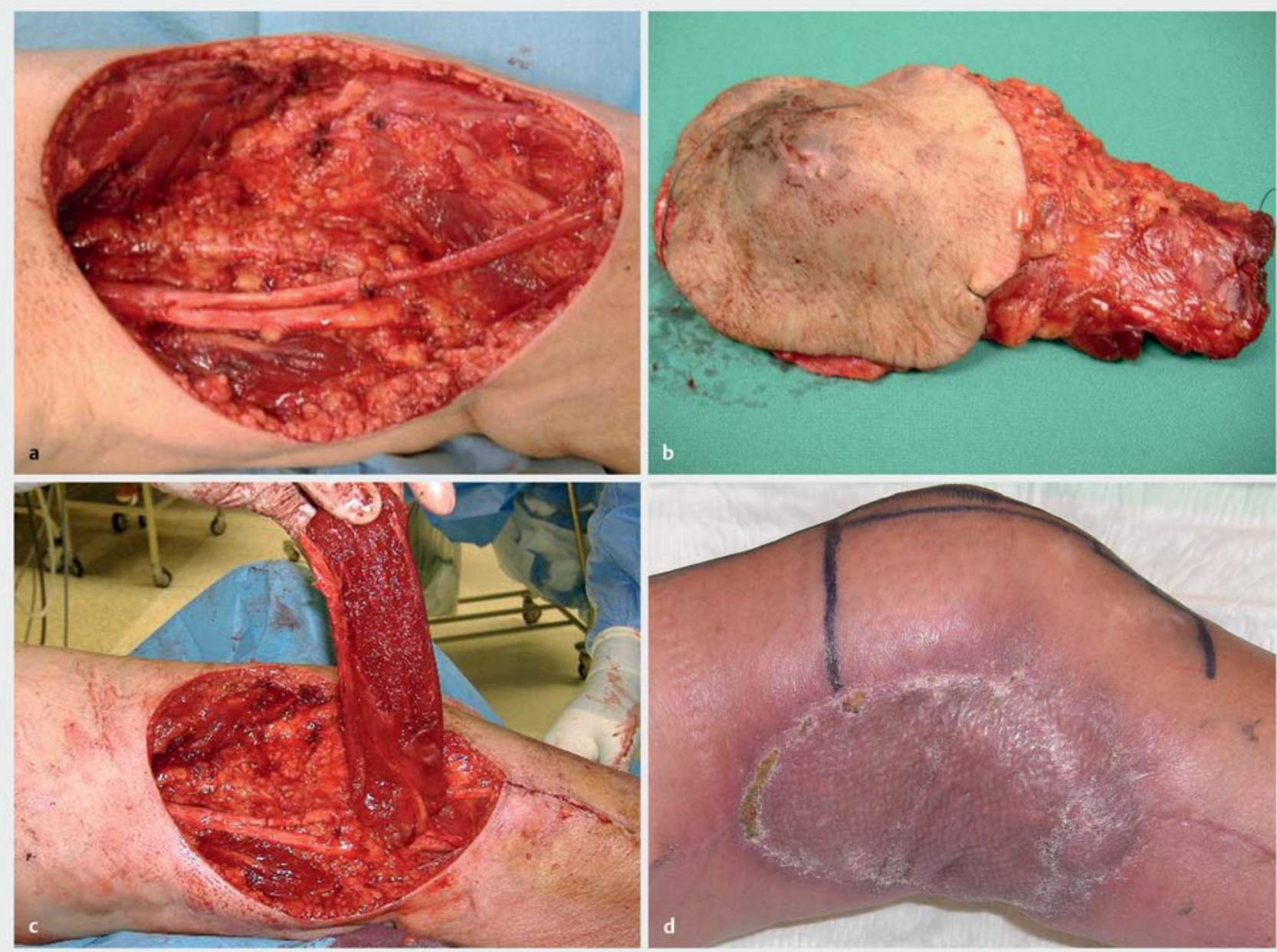

- Abb. 3 a Defekt nach Resektion eines pleomorphen Sarkoms im dorsolateralen Kniebereich. b Resektat des pleomorphen Sarkoms. c-d Rekonstruktion mit gestieltem lateralen Gastroknemiuslappen und Spalthaut.

Weitere Lappenplastiken um definierte Hautgefäße sind für die A. genicularis superior und die A. suralis beschrieben worden. Prinzipiell sind für diese Form der Lappenplastik aber auch um alle anderen nicht speziell benannten Gefäße geeignet, die in der Umgebung gefunden werden $[2,3]$.

\section{Gefäßgestielte Muskellappenplastiken}

Medialer und lateraler M.-gastrocnemius-Lappen

Der mediale und der laterale Bauch des M. gastrocnemius sind konstant hinsichtlich ihrer Anatomie und ihrer Gefäßversorgung. Die Muskellappen erreichen problemlos nach proximal den oberen Pol der Patella und nach distal den Tibiakopf. Die fehlenden Muskelteile können durch den M. soleus und den ggf. verbliebenen 2. Muskelbauch gut kompensiert werden. Durch den Muskel können auch größere Defekte gedeckt und tiefe Wundhöhlen gefüllt werden. Aufgrund dieser Voraussetzungen wird dieser Lappen seit 1978 mit Abstand am häufigsten zur Rekonstruktion von Defekten im Kniebereich verwendet [4].
Die Blutversorgung erfolgt ber die A. suralis aus der A. poplitea. Die beiden Muskelbäuche unterscheiden sich allerdings in einigen Punkten voneinander. Der mediale Gastroknemiusbauch ist länger und breiter. Der laterale Gastroknemiusbauch muss um die Fibula nach vorne gelegt werden, wodurch die verfügbare Länge noch einmal reduziert wird. Bei der Hebung des lateralen Muskelteiles ist auf die Schonung des N. peronaeus communis zu achten.

Die Hebung erfolgt durch einen medialen bzw. lateralen Längsschnitt, die Faszie über dem Muskel wird eröffnet. Das Septum zwischen M. gastrocnemius und soleus wird aufgesucht, zwischen diesen Muskeln verläuft die Sehne des M. plantaris. Dorsal zwischen den Muskelbäuchen liegt der N. suralis. Dieser muss vorsichtig vom Muskel gelöst werden und markiert die Mitte zwischen den beiden Muskelbäuchen. Diese werden voneinander getrennt und der Muskellappen distal von der Achillessehne abgesetzt. Bei Bedarf kann über eine Schnitterweiterung in die 


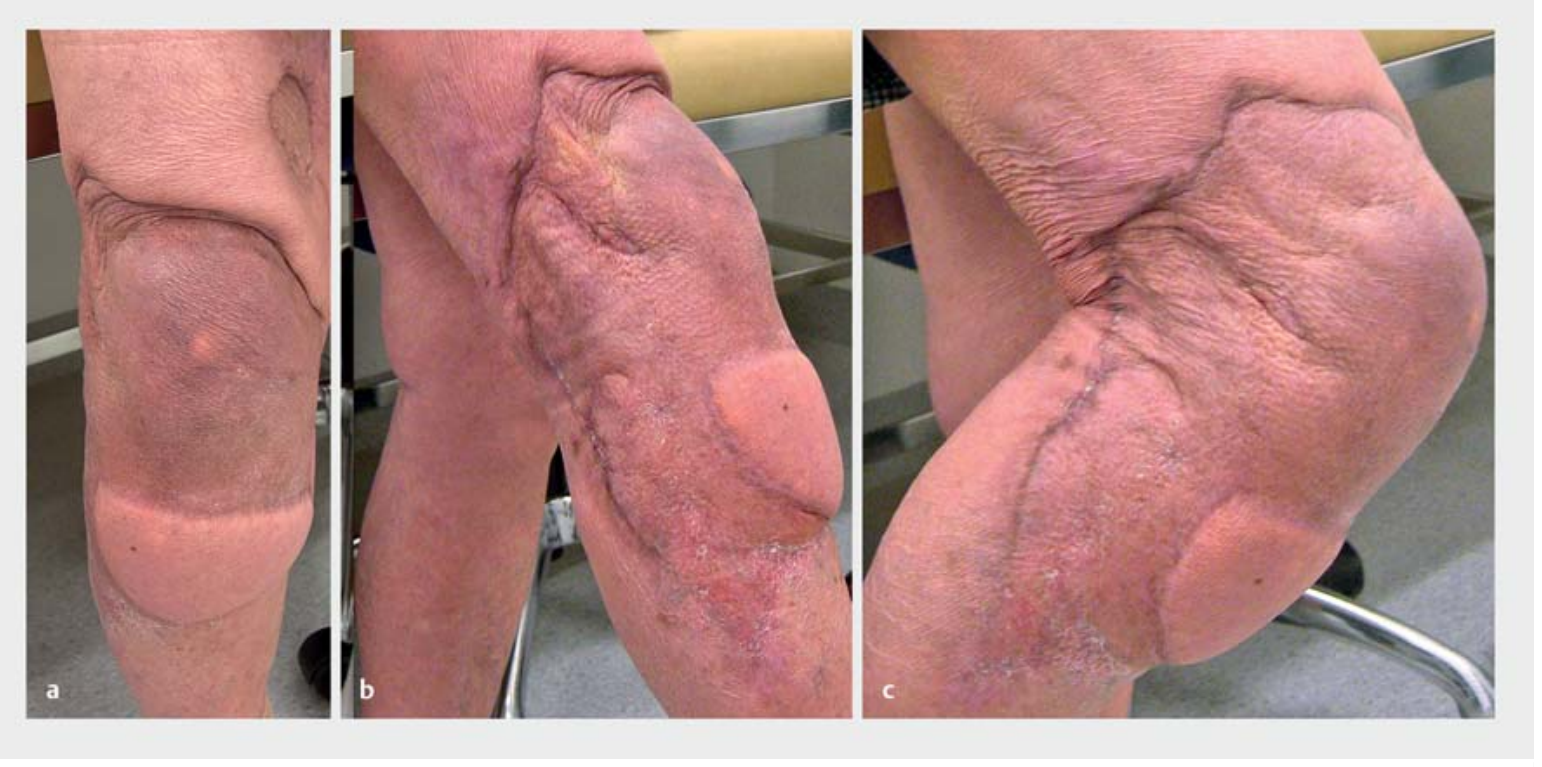

Abb. 4 Latissimuslappen im Kniebereich in Streckung und Beugung. Defekt durch Pyoderma gangraenosum nach Kniearthroskopie.

Kniekehle der Muskel auch vom Ansatz am Oberschenkel unter Erhalt des Gefäßstieles abgesetzt werden. Die sehnigen Anteile werden entfernt oder eingekerbt, um die Reichweite des Muskels zu erhöhen. Anschließend kann der Muskel in den Defekt eingelegt werden ( $\mathbf{A} \mathbf{b b}$. $\mathbf{3} \mathbf{a}-$ d).

Beschrieben ist eine gleichzeitige Rekonstruktion oder Augmentation des Lig. patellae durch die sehnigen distalen Anteile des Gastroknemiusmuskels [5].

\section{M.-soleus-Lappen}

Der M. soleus wurde ebenfalls bereits zur Rekonstruktion von distalen Kniedefekten verwendet. Aufgrund der deutlich schwierigeren Hebung, insbesondere aber aufgrund von dessen wichtiger Rolle bei der Muskelpumpe des tiefen Venensystems sollte anderen Lappenplastiken der Vorzug gegeben werden.

\section{M.-vastus-lateralis-Lappen, distal gestielt}

Basierend auf der bereits oben beschriebenen Anatomie des ALT-Lappens ist auch die Hebung eines Anteiles des Vastus-lateralis-Muskels mit einem distalen Gefäßstiel möglich. Auch hier liegt die Limitierung in der Inkonstanz einer ausreichenden distalen Anastomose [1].

\section{M.-sartorius-Lappen}

Der M. sartorius wird - im Unterschied zu den bisher beschriebenen Muskeln - nicht von einem dominanten Gefäß, sondern von mehreren segmentalen Gefäßen im gesamten Verlauf versorgt. Möglich ist hier eine Durchtrennung der proximalen Gefäße in einem ersten Schritt. Nach einem Intervall ist die neue Blutversorgung etab-

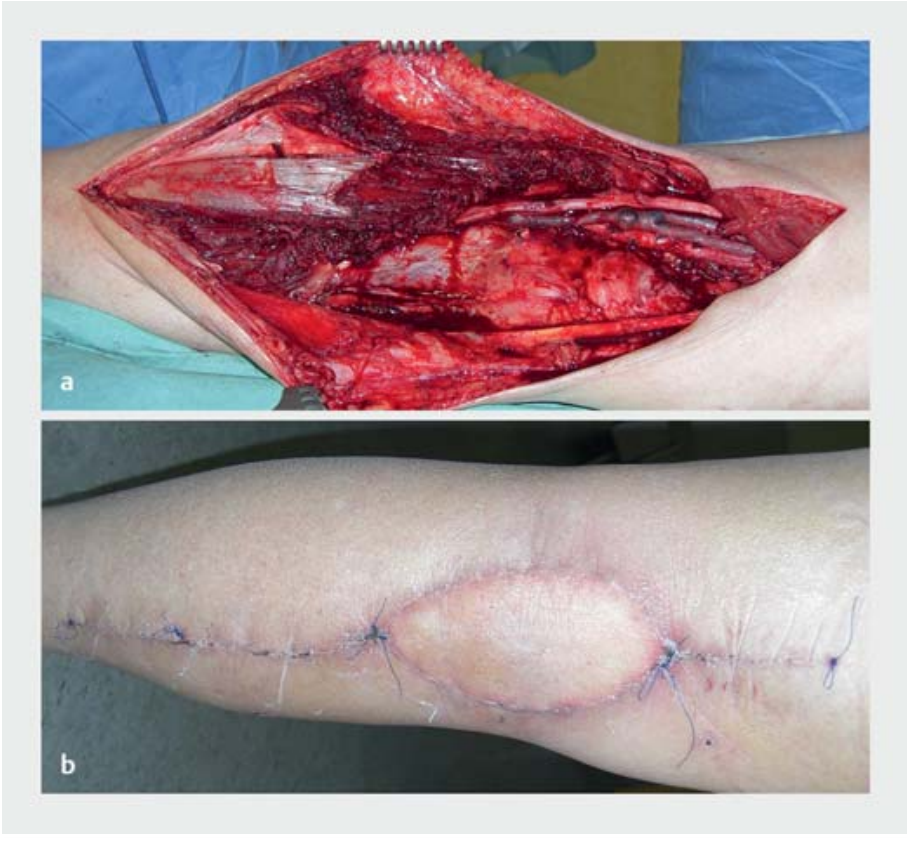

- Abb. 5 a Defekt in der Kniekehle nach Sarkomresektion. b Deckung in der Kniekehle mit freiem lateralen Oberarmlappen.

liert. Der Muskel kann proximal durchtrennt und nach distal umgelegt werden. Erreicht wird hiermit insbesondere der proximale Anteil des Kniegelenks. Er eignet sich für kleinere Defekte, insbesondere aber in Kombination mit dem medialen Gastroknemiuslappen bei größeren Defekten $[6,7]$. 


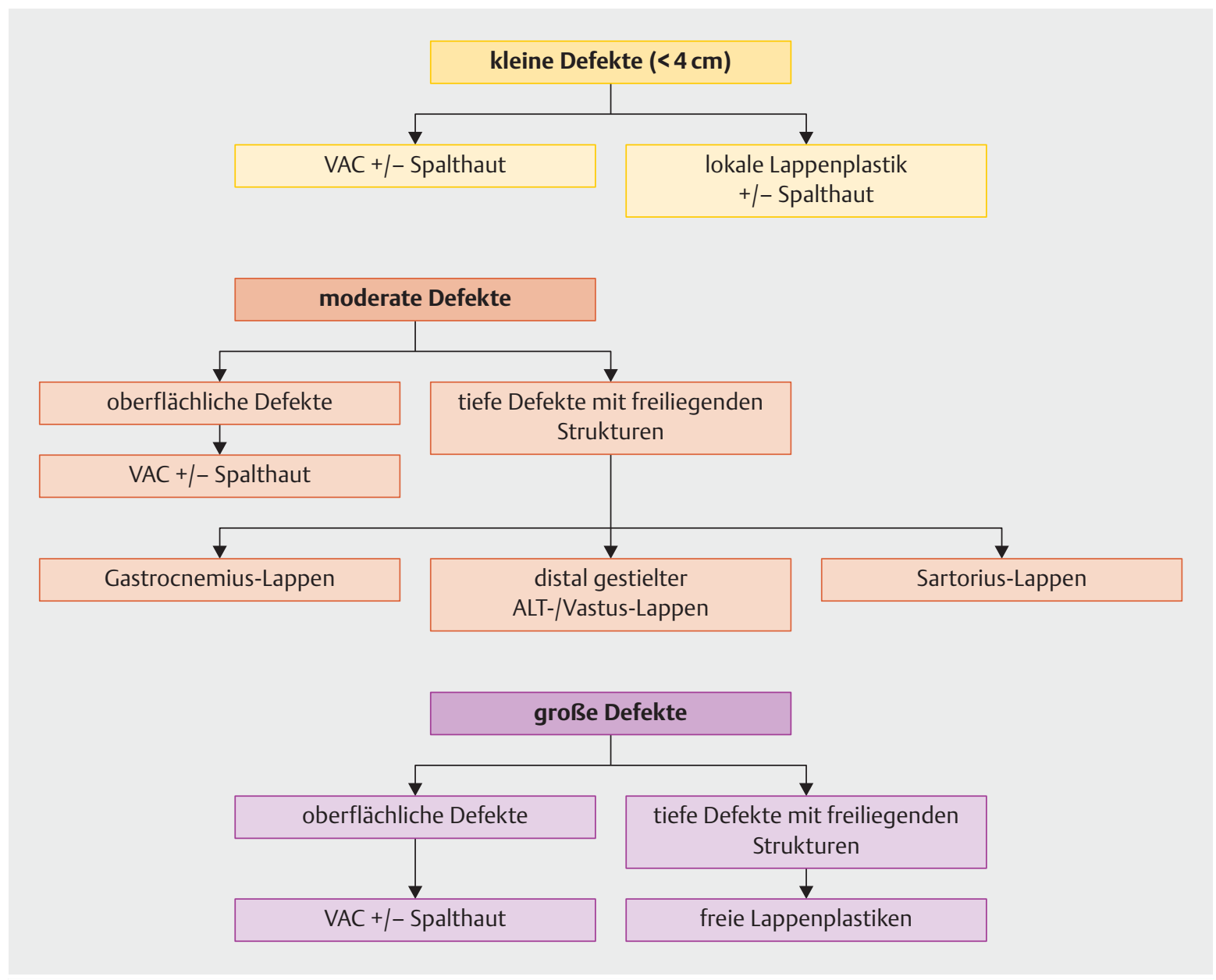

- Abb. 6 Algorithmus zur Defektdeckung nach Defektgröße.

\section{Freie Lappenplastiken}

Bei sehr ausgedehnten Defekten oder bei Defekten nach einer Tumorresektion können freie Lappenplastiken indiziert sein. Hierzu wird ein Lappen fern des Defektes mit einem definierten Gefäßstiel gehoben und die Blutversorgung durch eine arterielle und eine venöse mikrochirurgische Gefäßverbindung wiederhergestellt.

\section{Anschlussgefäße}

Eine wesentliche Voraussetzung für einen freien Gewebetransfer ist das Vorliegen von geeigneten Anschlussgefäßen. Diese finden sich auf Höhe des Kniegelenkes vor allem im Bereich der Kniekehle. Dies erleichtert den Transfer bei dorsalen Defekten, verlängert aber die Strecke zu einem ventralen Defekt wesentlich. Daher eignen sich hier vor allem Lappen mit einem langen Gefäßstiel.

Für einen Gefäßanschluss eignen sich neben der A. poplitea vor allem die Aa. superiores genus medialis und lateralis. Diese entspringen aus der A. poplitea im Bereich der Kniekehle und können hier gut dargestellt werden. Die übrigen Gefäße sind hier oftmals sehr klein und tief gelegen und damit zum Gefäßanschluss weniger geeignet.

Prinzipiell ist die Auswahl an freien Lappen mittlerweile sehr vielfältig geworden. Unter Berücksichtigung der limitierten Auswahl an Anschlussgefäßen und dem damit wünschenswert langen Gefäßstiel sowie der Indikation freier Lappen bei sehr ausgedehnten Defekten reduziert sich die Auswahl wieder deutlich.

\section{M.-latissimus-dorsi-Lappen}

Der M. latissimus dorsi ist sicher für ausgedehnte ventrale Defekte aus verschiedener Sicht gut geeignet. Er besitzt einen langen Gefäßstiel von bis zu $12 \mathrm{~cm}$. Die Größe des Muskels erlaubt die Deckung auch sehr ausgedehnter Defekte. Durch seine flächige Ausdehnung kann der Muskel dünn in den Defekt eingepasst werden ( $\bullet \mathbf{A b b}$. 4a und $\mathbf{b})$. Die Seitenlage bei der Operation erlaubt eine parallele Hebung des Muskels und eine Darstellung der Anschlussgefäße $[8,9]$. 


\section{M.-gracilis-Lappen}

Der Vorteil des Gracilismuskels liegt vor allem im geringen Hebedefekt [10]. Durch die Länge des Muskels kann, trotz des verhältnismäßig kurzen Gefäßstieles, auch das ventrale Kniegelenk erreicht werden. Die distale Gracilissehne kann zusätzlich als Ersatz für eine defekte Patellarsehne verwendet werden $[11,12]$.

\section{Anterolateraler Oberschenkellappen (ALT) und M.-vastus-lateralis-Lappen}

Bereits oben beschrieben ist der ALT-Lappen distal gestielt [1]. Dieser eignet sich sehr gut auch als freier Lappen bei ungenügender distaler Anastomose aufgrund seines langen Gefäßstieles. Mit dem gleichen Stielgefäß können auch Anteile des M. vastus lateralis gehoben werden. Dies eignet sich in besonderer Weise im Falle einer gleichzeitigen Kniegelenksarthrodese, da hierdurch kein funktionelles Defizit entsteht.

\section{Lateraler Oberarmlappen}

Der laterale Oberarmlappen eignet sich vor allem bei Defekten im Bereich der Kniekehle. Die gut dehnbare Haut ermöglicht hier eine Rekonstruktion ohne eine Beeinträchtigung der Kniestreckung ( $\bullet \mathbf{A b b} \mathbf{5} \mathbf{a}$ und b). Ein Vorteil ist die konstante Anatomie. Der Hebedefekt ist allerdings deutlich sichtbar $[13,14]$.

\section{Fazit}

Es gibt in der Literatur verschiedene Ansätze für einen Algorithmus der Defektdeckung im Kniebereich ( $\bullet$ Abb. 6). Unstrittig sind die Hauttransplantate bei oberflächlichen Defekten ebenso wie lokale Lappen bei kleineren (bis ca. $4 \mathrm{~cm}$ ) oberflächlichen Defekten und bei kontrakten Narben. Für größere Defekte im ventralen Bereich des Kniegelenkes ist der mediale und/oder laterale Gastroknemiuslappen etabliert. Bei sehr ausgedehnten Defekten ist der freie Latissimuslappen das Mittel der Wahl [15].

Es gibt nur relativ selten Defekte, bei denen o.g. Rekonstruktionen ungeeignet erscheinen. Mögliche Gründe hierfür sind vielfältig. So kann z. B. die Größe und Lage des Defektes, das Vorliegen einer arteriellen Verschlusskrankheit oder einer sehr kräftigen Subkutanschicht einen bestimmten Lappen eher geeignet oder ungeeignet erscheinen lassen. Ein zusätzlicher Defekt der Patellarsehne kann für die Verwendung eines Lappens mit einem Sehnenanteil zur Rekonstruktion sprechen. Nicht zuletzt spielt sicher auch die eigene Erfahrung des Operateurs bei der Auswahl eine nicht geringe Rolle. Damit bleibt in diesen Fällen die Entscheidung individuell und kann nur bedingt in einen festen Algorithmus gebracht werden.

\section{Interessenkonflikt}

Die Autoren geben an, dass kein Interessenkonflikt besteht.
Autorinnen/Autoren

\section{Stefan Krischak}

Dr. med., Sektionsleiter Plastische und Handchirurgie, Klinik für Unfallchirurgie, Orthopädie, Plastische und Handchirurgie, Universitätsklinikum Augsburg

\section{Matthias Föhn}

Dr. med., Klinik für Unfallchirurgie, Orthopädie, Plastische und Handchirurgie, Universitätsklinikum Augsburg

\section{Korrespondenzadresse}

\section{Dr. Stefan Krischak}

Klinik für Unfallchirurgie, Orthopädie,

Plastische und Handchirurgie

Universitätsklinikum Augsburg

Stenglinstraße 2

86156 Augsburg

stefan.krischak@klinikum-augsburg.de

\section{Literatur}

[1] Bekarev M, Goch AM, Geller DS et al. Distally based anterolateral thigh flap: an underutilized option for peri-patellar wound coverage. Strategies Trauma Limb Reconstr 2018; 13: 151162. doi:10.1007/s11751-018-0319-9

[2] Wiedner M, Koch H, Scharnagl E. The superior lateral genicular artery flap for soft-tissue reconstruction around the knee: clinical experience and review of the literature. Ann Plast Surg 2011; 66: 388-392

[3] Ling BM, Wettstein R, Staub D et al. The medial sural artery perforator flap: the first choice for soft-tissue reconstruction about the knee. J Bone Joint Surg Am 2018; 100: 211-217

[4] Feldman JJ, Cohen BE, May JW jr. The medial gastrocnemius myocutaneous flap. Plast Reconstr Surg 1978; 61: 531-539

[5] Fansa H, Plogmeier K, Schenk K et al. Die Deckung ausgedehnter Weichteildefekte bei infizierten Knieendoprothesen durch Gastrocnemiuslappen. Chirurg 1998; 69: 1238-1243

[6] Manjunath KN, Venkatesh MS, Shivaprasad A. Distal major pedicle of sartorius muscle flap: anatomical study and its clinical implications. Indian J Plast Surg 2018; 51: 40-45

[7] Bouveau V, Potage D, Dubory A et al. A distally based sartorius muscle flap for a Gustilo grade-III open fracture of the lateral femoral condyle and the head of the fibula with a complex soft-tissue defect: a case report and review of the literature. JBJS Case Connect 2017; 7: e93

[8] Fisher J, Cooney WP 3rd. Designing the latissimus dorsi free flap for knee coverage. Ann Plast Surg 1983; 11: 554-562

[9] Maghari A, Forootan KS, Fathi M et al. Free transfer of expanded parascapular, latissimus dorsi, and expander "capsule" flap for coverage of large lower-extremity soft-tissue defect. Plast Reconstr Surg 2000; 106: 402-405

[10] Besset M, Penaud A, Quignon R et al. [Donor site morbidity after free gracilis muscle flap. Report of 32 cases]. Ann Chir Plast Esthet 2014; 59: 53-60

[11] Macchi V, Vigato E, Porzionato A et al. The gracilis muscle and its use in clinical reconstruction: an anatomical, embryological, and radiological study. Clin Anat 2008; 21: 696-704 
[12] Nieminen H, Kuokkanen H, Tukiainen E et al. Free flap reconstructions of tibial fractures complicated after internal fixation. J Trauma 1995; 38: 660-664

[13] Altmann S, Damert HG. [The plastic coverage of soft-tissue defects after endoprosthesis on the lower limb]. Zentralbl Chir 2013; 138 (Suppl. 2): e36-e40

[14] Ulusal BG, Lin YT, Ulusal AE et al. Reconstruction of foot defects with free lateral arm fasciocutaneous flaps: analysis of fifty patients. Microsurgery 2005; 25: 581-588
[15] Gravvanis A, Kyriakopoulos A, Kateros K et al. Flap reconstruction of the knee: a review of current concepts and a proposed algorithm. World J Orthop 2014; 5: 603-613

\section{Bibliografie}

DOI https://doi.org/10.1055/a-0785-4618

Online-publiziert 22.02.2019 | OP-JOURNAL 2019; 35: 172180 @ Georg Thieme Verlag KG Stuttgart · New York ISSN 0178-1715 